\title{
Thermally-Compensated Magnetic Core Loss Model for Time-Domain Simulations of Electrical Circuits
}

N. Djekanovic, M. Luo, and D. Dujic

This material is posted here with permission of the IEEE. Such permission of the IEEE does not in any way imply IEEE endorsement of any of EPFL's products or services. Internal or personal use of this material is permitted. However, permission to reprint / republish this material for advertising or promotional purposes or for creating new collective works for resale or redistribution must be obtained from the IEEE by writing to pubs-permissions@ieee. org. By choosing to view this document, you agree to all provisions of the copyright laws protecting it. 


\title{
Thermally-Compensated Magnetic Core Loss Model for Time-Domain Simulations of Electrical Circuits
}

\author{
Nikolina Djekanovic, Student Member, IEEE, Min Luo, Member, IEEE, and \\ Drazen Dujic, Senior Member, IEEE
}

\begin{abstract}
This paper introduces a thermally-compensated magnetic hysteresis model, capable of accurately determining core losses of ferrite materials while accounting for core temperature variations. The employed model is based on permeancecapacitance analogy and it captures the frequency-independent hysteresis effect with the help of the Preisach model. For validation purposes, a $100 \mathrm{~kW}, 10 \mathrm{kHz}$ realized medium frequency transformer prototype is selected and modeled as a part of a full-bridge LLC resonant converter in a time-domain simulation environment. Conducted simulations show the ability of the thermally-compensated magnetic hysteresis model to impact the generated core losses for core temperatures of up to $120{ }^{\circ} \mathrm{C}$. Finally, a temperature-feedback loop within the transformer is successfully closed inside of a system-level simulation, allowing for a more precise determination of the core and winding steadystate temperatures.
\end{abstract}

Index Terms-Resonant LLC Converter, Medium Frequency Transformer, Hysteresis Model, Thermal Compensation, TimeDomain Simulation

\section{INTRODUCTION}

A temperature rise caused by generation of various losses during a power converter operation impacts not only the environment, but all the converter components as well. This in turn influences the overall performance of the converter and it is an effect which needs to be adequately considered. When designing a power converter system in general, it is essential to know the thermal constraints of the components to avoid damage and overheating, but also to help in properly sizing the cooling system. Moreover, by understanding thermal limits of the key converter elements, such as switching devices and magnetic components, it is possible to improve the overall power efficiency. For this purpose, advanced design tools are necessary, which are capable of simulating various relevant power converter domains at the same time in a common simulation environment. Such domains include electrical, magnetic, dielectric and thermal domain. Commonly used system-level simulation tools are capable of determining different types of losses arising from power semiconductor devices. In addition, they can also provide accurate predictions of the junction temperature of the device and take it into account for loss determination. Thanks to the availability of standardized data sheets which contain important technical information of the device, such as rated values and switching characteristics,

Nikolina Djekanovic and Drazen Dujic are with the Power Electronics Laboratory, EPFL, 1015 Lausanne, Switzerland (e-mail: nikolina.djekanovic@epfl.ch; drazen.dujic@epfl.ch).

Min Luo is with Plexim GmbH, 8005 Zurich, Switzerland (e-mail luo@plexim.com). power electronics designers are able to predict behavior of the semiconductor device to a reliable degree, without having to perform additional tests.

Nevertheless, when it comes to standard magnetic components, such as filter inductors and transformers, the loss determination is not as straightforward and there is generally still no standardized approach. The corresponding data sheets mostly offer coarse information about the expected losses, and only the data sheets of magnetic core materials provide additional data, which can be used for loss estimation. Another existing barrier is the fact that manufacturers usually characterize the core material losses by using sinusoidal excitation, which is different to operating conditions usually found in state-of-theart converter topologies (such as the Resonant LLC Converter [1] and the Dual Active Bridge [2]).

According to [3], magnetic core losses can be principally divided into three parts. Namely, frequency-independent hysteresis loss, frequency-dependent eddy current loss and relaxation loss. To accurately model the magnetic hysteresis phenomenon different models have been suggested in the past. Many of them rest on nonlinear hysteresis characterization achieved through pure mathematical models which do not consider the underlying physics of the material behaviour [4], [5]. On the other hand, two main macroscopic hysteresis models which are based on actual physical assumptions are: the more complex Jiles-Atherton (J-A) model [6], [7] and the Preisach model [8]. The first model considers the hysteresis effect as a consequence of frictional forces opposing the motion of the Bloch domain-wall [9], whereas the latter model is specific due to its phenomenological character. This implies that the model is able to reproduce the hysteresis curve and the corresponding magnetic field history with great accuracy and reliability without explaining the physical processes occurring during the magnetization of the material [10]. Moreover, both models are capable of accurate hysteresis modeling under different operating conditions, i.e. for various excitation voltages. However, in respect to modeling of minor loops the Preisach model, in contrast to J-A model, is mathematically more stable [11]. In addition, the Chan-Vladimirescu model [12] is used for core materials with symmetrical magnetization process, due to its simplicity. The three models are frequently employed for time-domain simulation purposes and have been implemented in finite element simulation tools.

Concerning technical applications in power electronics field, ferrites are one of the most important magnetic materials. They are widely used for producing magnetic cores for inductive components such as chokes, filters, and transformers. Yet, 
numerous studies show that different ambient conditions have an effect on the properties of magnetic materials in varying degrees [13]-[15], among which the temperature is the key impact factor. Particularly in case of ferrites, the conductivity of the material increases with the rise of temperature, whereas the permeability in the linear region of the magnetization curve remains unaffected. Note that for other types of magnetic materials ( $\mathrm{Fe}-\mathrm{Si}$, nanocrystalline, amorphous metal) increased temperature exhibits its influence in a different manner [16]. As a consequence, the core losses are significantly impacted. Nevertheless, the exact temperature impact is often ignored in the existing works which deal with core-loss modeling. Therefore, this paper deals particularly with the temperature effect and the influence it has on the ferrite-core losses, when such a magnetic component is a part of a larger power converter system. For those purposes, the resonant LLC converter is selected as a case study topology and simulated with the help of electrical circuit simulation tool PLECS. Thereby, the idea is to demonstrate the temperature effect on the ferrite transformer losses and to introduce a magnetic hysteresis model which is able to incorporate temperature as an additional input to the model. As an application example of a magnetic component, a medium frequency transformer (MFT) prototype developed in [17], which is used for galvanic isolation in the LLC converter, is simulated and studied in detail. The thermally-compensated magnetic-hysteresis model is based on a recently developed magnetic model [11], [18], which is used as a starting point for accurate representation of the hysteresis effect within the transformer core and it is implemented with the help of the Preisach model. Note that the followed modeling approach is not restricted to the selected simulation tool. The simulated core temperature is one of the outputs of a simplified thermal MFT model, initially developed in [19] for the selected transformer prototype.

Key novelties and contributions of this paper are: 1) a thermally-compensated magnetic hysteresis model able to estimate core losses of ferrite components in a temperature range of $\left[20^{\circ} \mathrm{C}, 120^{\circ} \mathrm{C}\right]$, based on a limited set of hysteresis loops measured at different temperatures; 2) a closed temperature feedback loop of a transformer inside of a simulation, which considers both core and winding losses as heat sources; 3 ) an accurate determination of the steady-state temperature of the core and windings; 4) a single time-domain simulation combining three key domains of a power converter, namely, electrical, magnetic, and thermal domain.

This paper is organized as follows: Section II introduces the case study selected to demonstrate the functionality of the suggested magnetic model. Section III provides details about the thermally-compensated magnetic model and explains the model implementation based on thermal characterization of the core material. In addition, the winding-loss model and its thermal extension are included as well. In Section IV, the simplified thermal model of the transformer is introduced. Section V discusses and compares the simulated converter losses with and without thermal compensation of the winding and the magnetic hysteresis model. Lastly, Section VI highlights the main findings of the presented work.

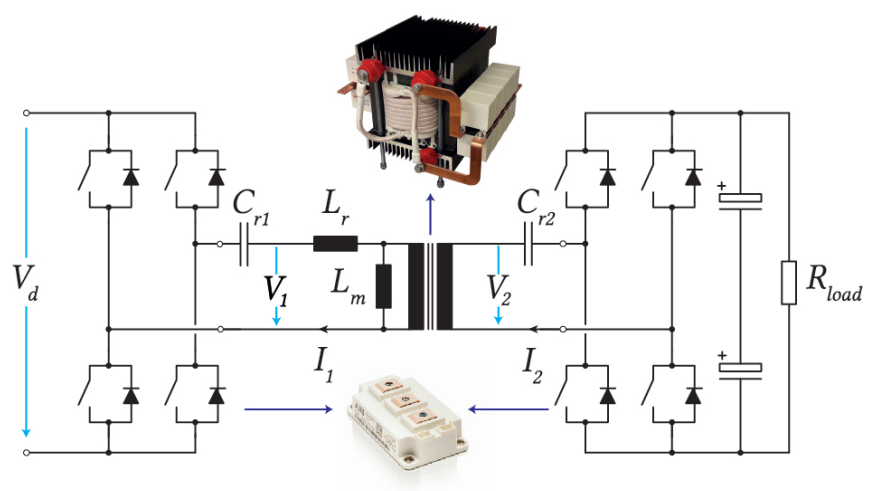

Fig. 1. Electrical circuit of the resonant LLC converter used for the simulation with a graphical preview of the MFT and the switching device.

\section{CAse Study: Resonant LlC Converter}

To demonstrate the possibility of thermal compensation of the magnetic hysteresis model with the help of simulations, a full-bridge resonant LLC converter is chosen as an exemplary topology for a power converter system. It comprises two fullbridge power stages, interlinked by an MFT with mounted resonant capacitors (labeled with $C_{\mathrm{r} 1}$ and $C_{\mathrm{r} 2}$ ), as can be seen in Fig. 1. The two inductor components of the resonant tank are integrated in the transformer, which is used for galvanic isolation of the converter. Namely, the transformer leakage and the magnetizing inductance are deployed as a series $\left(L_{\mathrm{r}}\right)$ and a shunt inductor $\left(L_{\mathrm{m}}\right)$, respectively. The model of the MFT is based on specifications of a $100 \mathrm{~kW}, 10 \mathrm{kHz}$ transformer prototype realized in [17]. The transformer core was constructed from $48 \mathrm{U}$-shaped magnetic core samples, made of ferrite material grade CF139. The first power stage is supplied with $750 \mathrm{~V}$ dc-link, whereas the second stage is used as a diode rectifier, since the power is transferred in a single direction. Table I summarizes the exact electrical specifications of the considered LLC converter. The resonant capacitor bank was realized as a series connection of multiple parallel ac film capacitors accounting to $37.5 \mu \mathrm{F}$, placed on each side of the MFT. With unity turns ratio of the transformer (each winding consists of eight turns), this leads to an equivalent resonant capacitance of $18.75 \mu \mathrm{F}$.

One of the main advantages of the LLC converter are the low switching losses, achieved through zero-voltage switching (ZVS) [1]. Considering a sub-resonant switching frequency,

TABLE I: Electrical specifications of the simulated LLC converter.

\begin{tabular}{lccc}
\hline Electric Property & Label & Unit & Value \\
\hline Rated Power & $P_{\mathrm{n}}$ & $\mathrm{kW}$ & 100 \\
Input Voltage & $V_{\mathrm{d}}$ & $\mathrm{V}$ & 750 \\
Output Voltage & $V_{\mathrm{o}}$ & $\mathrm{V}$ & 750 \\
Switching Frequency & $f_{\mathrm{sw}}$ & $\mathrm{kHz}$ & 10 \\
Duty Cycle & $D$ & $\mathrm{p} . \mathrm{u}$. & 0.5 \\
Leakage Inductance & $L_{\mathrm{r}}$ & $\mu \mathrm{H}$ & 8.4 \\
Magnetizing Inductance & $L_{\mathrm{m}}$ & $\mu \mathrm{H}$ & 750 \\
Resonant Capacitor & $C_{\mathrm{r}}$ & $\mu \mathrm{F}$ & 18.75 \\
\hline
\end{tabular}


which is set based on two characteristic resonant frequencies related to the resonant tank of the converter

$$
\begin{aligned}
& f_{0}=\frac{1}{2 \pi \sqrt{L_{\mathrm{r}} C_{\mathrm{r}}}}=12.68 \mathrm{kHz}, \\
& f_{\mathrm{p}}=\frac{1}{2 \pi \sqrt{\left(L_{\mathrm{r}}+L_{\mathrm{m}}\right) C_{\mathrm{r}}}}=1.33 \mathrm{kHz},
\end{aligned}
$$

a half-cycle discontinuous conduction mode (HC-DCM) is achieved, which allows soft commutation of the rectifier diodes in the secondary stage. The higher resonant frequency $f_{0}$ is the one of interest for the control and operating characteristics of the converter. The primary and secondary current waveforms are shown in Section V.

\section{Modeling OF the Resonant LLC CONVERTER LOSSES}

\section{A. Magnetic Core Modeling and Losses}

Due to the low electrical conductivity of ferrite materials at temperatures of up to $100^{\circ} \mathrm{C}$, it is safe to claim that the eddy current losses can be neglected [18]. Furthermore, the relaxation losses are mostly present in high-frequency applications above $20 \mathrm{kHz}$ and when voltage waveforms with zero phase voltages are applied to the magnetic component [20]. Both the former and the latter do not apply to an MFT operated within an LLC converter in the selected case study. Therefore, the frequency-independent hysteresis effect is considered to be the dominant core loss effect for the studied transformer prototype.

The magnetic model developed in [11] and used as a basis for modeling of the MFT prototype rests on the permeancecapacitance analogy. According to this, any magnetic core shape can be represented by a circuit of permeances

$$
\mathscr{P}=\mu \frac{A}{l} .
$$

Thereby, the parameters $A$ and $l$ describe the geometry of a specific core part, namely its cross section area and the corresponding magnetic path length (MPL). The permeability $\mu$ reflects the nonlinear nature of the core material, i.e. the hysteresis phenomenon, and within the classical model of Preisach [8] it is modeled as a function of the field strength $(\mu(H))$. The followed modeling approach is valid for frequencies up to



Fig. 2. Transformer core represented by a set of permeances in a magnetic circuit. Figure is adopted from [21].



Fig. 3. Magnetic characterization setup [11] for the set of U-cores made of ferrite material CF139.

$50 \mathrm{kHz}$ [18]. Fig. 2 shows the magnetic circuit corresponding to the model implementation of the MFT prototype in the simulation. A precise knowledge of the core geometry of a certain core part $(A, l)$ is essential and this needs to be adjusted for each permeance block in order to follow the overall MFT construction geometry. Now, the only missing information for correct parametrization of the permeance block is modeling of the nonlinear permeability. This is performed with the help of hysteresis measurements collected from a set of U-cores made of the same ferrite material CF139, which is used for the core of the MFT prototype. Thereby, a characterization setup with the structure proposed by [22] is employed, as shown in Fig. 3. The primary winding is excited by the power stage, whereas the secondary winding is left open for voltage measuring. Subsequently, the collected current and voltage measurements are converted to field strength $(H)$ and flux density $(B)$ inside the control unit through the following equations:

$$
H(t)=\frac{N_{1} i(t)}{l}, \quad B(t)=\frac{1}{N_{2} A} \int v(t) d t .
$$

The parameters $N_{1}$ and $N_{2}$ are the number of turns of the primary and the secondary winding, which can be adapted to obtain the desired $H$ and $B$ values on the hysteresis plane.

According to the formulation of the Preisach model the magnetic hysteresis is comprised of many smaller particles, known as hysterons $\gamma(U, V)$. Depending on the value of the applied magnetic field $H$, each of the square-loop hysterons switch between -1 and +1 at a set of boundary transition values $U$ and $V$, as can be seen in Fig. 4. Following this, the flux density can be represented as the weighted sum of all hysterons with a certain type of probability distribution function (PDF) $p(U, V)$ as the weight [11]:

$$
\begin{aligned}
B(H) & =\iint p(U, V) \cdot \gamma(U, V) \cdot d U d V \\
& =\iint p_{s}(U) \cdot p_{s}(-V) \cdot \gamma(U, V) \cdot d U d V
\end{aligned}
$$

Due to the fact that in case of soft-magnetic materials, such as ferrites, probabilities of a hysteron switching in one or the opposite direction are independent, $p(U, V)$ can be expressed as a product of two one-dimensional PDFs $p_{s}(H)$ [23]. For 


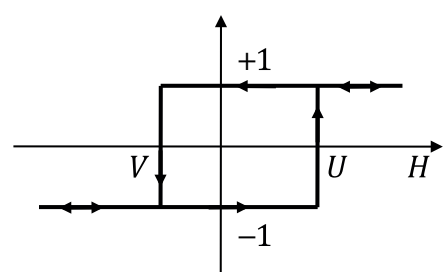

Fig. 4. A single hysteron $\gamma(U, V)$ [11].

the deployed magnetic model the logistic PDF is selected, in contrast to commonly used Cauchy-Lorentz PDF [11]. Nevertheless, both PDFs can be analytically integrated to closed forms. Eventually the nonlinear permeability is calculated as $\mu(H)=d B / d H$ and the exact calculation process depends on the position of the operating point on the B-H curve and the previous field strength extremity.

For correct model parametrization, it is sufficient to experimentally measure only two hysteresis loops, known as the limiting and the minor hysteresis loop. Due to current and voltage limitations of the characterization setup ( $24 \mathrm{~V}$ output voltage and $6 \mathrm{~A}$ output current), the maximal flux density of the first hysteresis loop does not reach real material saturation, but it marks the upper operation boundary of model validity. Fig. 5 shows the curves measured at room temperature of $20^{\circ} \mathrm{C}$ of the core. For the limiting hysteresis loop (measured at $\hat{H}_{100 \%}=100 \mathrm{~A} \mathrm{~m}^{-1}$ ) given in Fig. 5a necessary parameters for the parametrization are: $B_{r, 100 \%}^{*}$ and $\hat{B}_{100 \%}^{*}$, the remanence and the saturation flux density values, respectively, and $\hat{\mu}_{100 \%, \uparrow}^{*}$, which is the ascending permeability measured at the saturation value of the magnetic field $\hat{H}_{100 \%}^{*}$. For the symmetrical minor hysteresis loop (measured at $\hat{H}_{20 \%}=20 \mathrm{~A} \mathrm{~m}^{-1}$ ) visualized in Fig. 5b, the needed parameters are: $B_{r, 20 \%}^{*}$ and $\hat{B}_{20 \%}^{*}$, which are the remanence and the maximal measured flux density values of the minor loop. The extracted values are further referred to as the primary set of parameters. The asterisk sign marks the measured values.

According to [11], the hysteresis model is comprised of an irreversible and reversible component, since reversible magnetization is present in ferrite materials. The briefly introduced Preisach model can be used only for the irreversible part, whereas the reversible part, which is manifested by a singlelined hysteresis characteristic without any remanence flux density, is obtained by integrating a shifted arctan function. For the irreversible component, the logistic PDF is expressed as

$$
p_{s}(H)=K \cdot \frac{e^{-\left(H-H_{0}\right) \cdot \sigma}}{\left(1+e^{\left.-\left(H-H_{0}\right) \cdot \sigma\right)}\right.},
$$

with parameters $K, \sigma$, and $H_{0}$ which are to be determined. Due to specific manufacturing process of ferrites (no fieldannealing treatment), it can be assumed that $H_{0}=0$, which leads to a symmetrical PDF around the vertical axis. The two remaining parameters are determined by equating the extracted primary set of parameter values from the measured loops with the derived closed expressions for the irreversible permeability for various parts of the hysteresis curve (ascending, descending, virgin curve and others). More precise formulation and exact identification of the parameters for this component

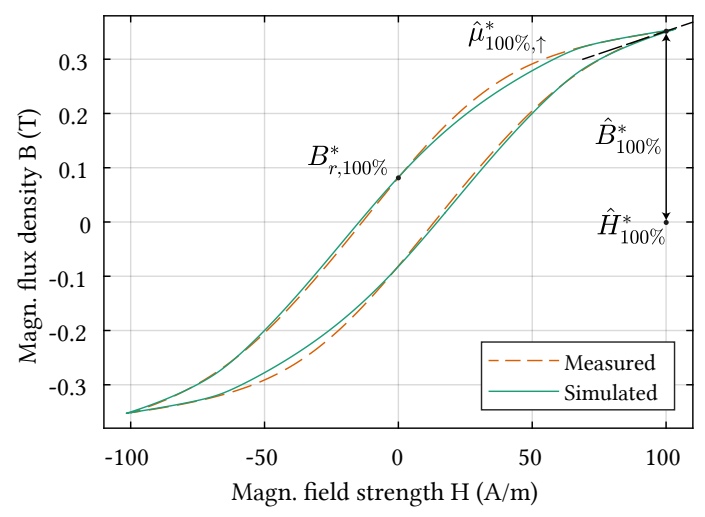

(a)

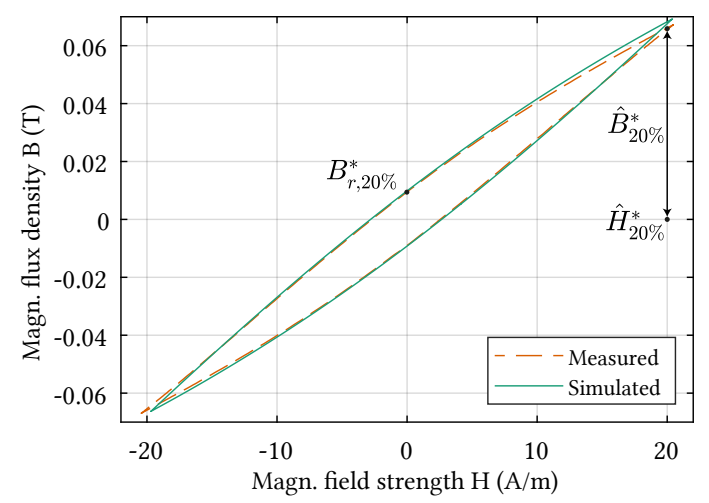

(b)

Fig. 5. Measured and simulated (a) limiting hysteresis loop with an amplitude of $100 \mathrm{~A} \mathrm{~m}^{-1}$ and (b) minor loop with an amplitude of $20 \mathrm{~A} \mathrm{~m}^{-1}$.

is presented in more detail in [11]. When determining the reversible component, the permeability can be expressed as following

$$
\mu^{\mathrm{rev}}(H)=F \cdot \arctan \left(\left(H_{1}-H\right) \cdot \alpha\right)+D,
$$

with $\alpha$ as the curvature parameter, which is manually set to improve the matching between the simulated and the measured hysteresis curve. The three unknown parameters $F, H_{1}$, and $D$ are again determined based on the measured primary set of parameters with the help of the Newton-Raphson iteration algorithm [11]. The implementation of the two permeability components in the simulation is realized with C-scripts.

Eventually, the matchings between the measured and the simulated limiting and minor hysteresis loops are also visualized in Fig. 5. Note that the two hysteresis curves are measured at $350 \mathrm{~Hz}$ with sinusoidal excitation. Nevertheless, due to the frequency-independent nature of the hysteresis loss effect, it is possible to accurately model hysteresis loops at higher operating frequencies. Finally, due to the physicalbased intrinsic property of the Preisach model, it can be assumed that the hysteresis loops with amplitudes between the measured limiting and the minor loop can be automatically approximated [21].

Analytical Core Losses: After performed core material characterization and the subsequent model parametrization a functional permeance block modeling the frequency-independent hysteresis effect is obtained. Eventually, the core losses are 
obtained by subtracting the power simulated at the secondary side from the primary side of the transformer. To verify the simulated losses an analytical approach is selected, based on a modification of the Steinmetz equation (SE). Compared to the original SE [24] which estimates the average power loss per volume unit

$$
P_{\mathrm{c}}=K f_{\mathrm{sw}}^{\alpha} B_{\mathrm{m}}^{\beta},
$$

the Improved Generalized SE (IGSE) provides higher estimation accuracy in the case of non-sinusoidal excitation. Thereby, $K, \alpha$ and $\beta$ are known as the Steinmetz loss coefficients. They are determined based on the core loss graphs provided in the core material data sheets depending on the flux density amplitude $B_{\mathrm{m}}$ and the excitation frequency $f_{\mathrm{sw}}$. According to [25], the IGSE is given by the following relation

$$
P_{\mathrm{c}}=\frac{1}{T} \int_{0}^{T} k_{\mathrm{i}}\left|\frac{d B(t)}{d t}\right|^{\alpha}(\Delta B)^{\beta-\alpha} d t,
$$

where $\Delta B$ is peak-to-peak flux density $\left(\Delta B=2 B_{\mathrm{m}}\right)$ and the coefficient $k_{i}$ is determined by

$$
k_{\mathrm{i}}=\frac{K}{2^{\beta+1} \pi^{\alpha-1}\left(0.2761+\frac{1.7061}{\alpha+1.354}\right)} .
$$

By considering a characteristic flux-density waveform for an resonant LLC converter and combining (9) and (10), the following equation for the average power loss per unit of volume is derived

$$
P_{\mathrm{c}}=2^{\beta} k_{\mathrm{i}} f_{\mathrm{sw}}^{\alpha} B_{\mathrm{m}}^{\beta}\left[D^{1-\alpha}+(1-D)^{1-\alpha}\right] .
$$

Parameters $D$ and $f_{\mathrm{sw}}$ are stated in Table I, whereas the flux density amplitude $B_{\mathrm{m}}$ is determined from a simulation. The analytical IGSE provides a single core loss value due to the fact that the core losses are independent from the loading conditions of the magnetic component. Eventually, the core losses simulated with the magnetic model for various power levels and the analytically obtained value for the selected MFT design and its operating characteristics at room temperature are given in Table II. Note that the variable $P_{n}$ stands for the rated power of the simulated LLC converter, given in Table I. A clear agreement between the simulation and analytics is visible. Nevertheless, a slight decreasing trend of the simulated core loss values is observed. This is explained by the primary transformer voltage $V_{1}$ variation depending on the operating point, which causes the magnetic model to reproduce a slightly altered hysteresis loop. The change in the primary voltage is due to the capacitor bank voltage $V_{C r 1}$. Due to lack of space, the corresponding simulated voltage and current waveforms are given in [26]. Conclusively, the core losses simulated with the magnetic hysteresis model match with the analytically obtained value. However, at this point it is not possible to explicitly consider the temperature influence on the ferrite material and its losses.

TABLE II: Core losses acquired through simulations at $10 \mathrm{kHz}$ for variable output powers and with the analytical equation, at a core temperature of $20^{\circ} \mathrm{C}$

\begin{tabular}{cccccccc}
\hline$P_{\mathrm{n}}$ & $0 \%$ & $20 \%$ & $40 \%$ & $60 \%$ & $80 \%$ & $100 \%$ & IGSE \\
\hline$P_{\text {core }}(\mathrm{W})$ & 229.6 & 226.4 & 223.8 & 221.2 & 218.6 & 216.1 & 225 \\
\hline
\end{tabular}

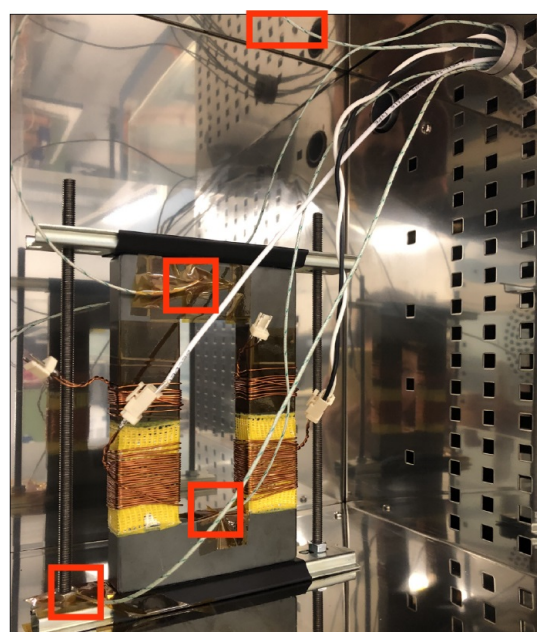

Fig. 6. Set of U-cores made of ferrite material CF139 inside of a temperature regulated oven, equipped with thermocouples.

\section{B. Thermal Extension of the Core Loss Model}

So far, there have been different approaches in accounting for thermal influence on magnetic materials and the corresponding core losses. In [27], this influence is considered for a typical operation temperature range $\left[20^{\circ} \mathrm{C}, 100^{\circ} \mathrm{C}\right]$, where the saturation points $B_{\text {sat }}$ and $H_{\text {sat }}$ change linearly with elevated temperatures, with the help of normalized limiting hysteresis loops. In [28], temperature and saturation coefficients are added to the Modified SE (MSE) [29] and Generalized SE (GSE) developed in [30] and experimentally verified in order to analytically model the temperature effect.

In this paper, the thermal effect is incorporated into the existing magnetic hysteresis model based on thermal characterization of the core material. This includes heating the set of $\mathrm{U}$-cores in a forced convection electric oven (Digitronic TFT Oven $250{ }^{\circ} \mathrm{C}$ DJ $250-47-\mathrm{V}$ ) to a certain set of temperatures and measuring each of the obtained hysteresis loops with the help of the magnetic characterization setup shown in Fig. 3. In order to reach a uniform temperature of the core material and the surrounding before a hysteresis measurement is performed, a set of thermocouples are placed at different parts of the U-set (bottom and top), at the clamp and one is let freely in the oven, which can be observed in Fig. 6 . Before each of the measurements were taken, it was made

TABLE III: Permeance block parameters extracted for different temperatures in the range of $\left[20^{\circ} \mathrm{C}, 120^{\circ} \mathrm{C}\right]$ together with per-cycle energy deviations between the measured and the simulated hysteresis loops.

\begin{tabular}{cccccccc}
\hline Temp. & $B_{r, 100 \%}^{*}$ & $\hat{B}_{100 \%}^{*}$ & $\hat{\mu}_{100 \%, \uparrow}^{*}$ & $B_{r, 20 \%}^{*}$ & $\hat{B}_{20 \%}^{*}$ & $\alpha$ & Dev. \\
\hline $20^{\circ} \mathrm{C}$ & $81.4 \mathrm{mT}$ & $352 \mathrm{mT}$ & 1526.6 & $9.4 \mathrm{mT}$ & $67.3 \mathrm{mT}$ & 0.2 & $-3.1 \%$ \\
\hline $40^{\circ} \mathrm{C}$ & $68.2 \mathrm{mT}$ & $350 \mathrm{mT}$ & 1245.2 & $8.3 \mathrm{mT}$ & $70.8 \mathrm{mT}$ & 0.12 & $-1.8 \%$ \\
\hline $60^{\circ} \mathrm{C}$ & $53.2 \mathrm{mT}$ & $344 \mathrm{mT}$ & 1130.9 & $6.3 \mathrm{mT}$ & $77.3 \mathrm{mT}$ & 0.16 & $0 \%$ \\
\hline $80^{\circ} \mathrm{C}$ & $40.7 \mathrm{mT}$ & $334 \mathrm{mT}$ & 1023.5 & $4.8 \mathrm{mT}$ & $82 \mathrm{mT}$ & 0.15 & $2.6 \%$ \\
\hline $100^{\circ} \mathrm{C}$ & $34.1 \mathrm{mT}$ & $322 \mathrm{mT}$ & 921.6 & $3.6 \mathrm{mT}$ & $82.5 \mathrm{mT}$ & 0.04 & $-0.3 \%$ \\
\hline $120^{\circ} \mathrm{C}$ & $35 \mathrm{mT}$ & $304 \mathrm{mT}$ & 759 & $3.7 \mathrm{mT}$ & $84 \mathrm{mT}$ & 0.05 & $-6.6 \%$ \\
\hline
\end{tabular}




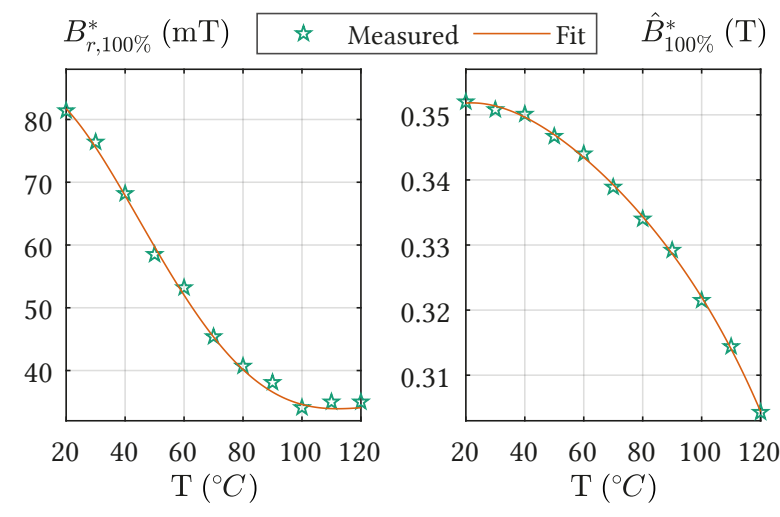

(a)
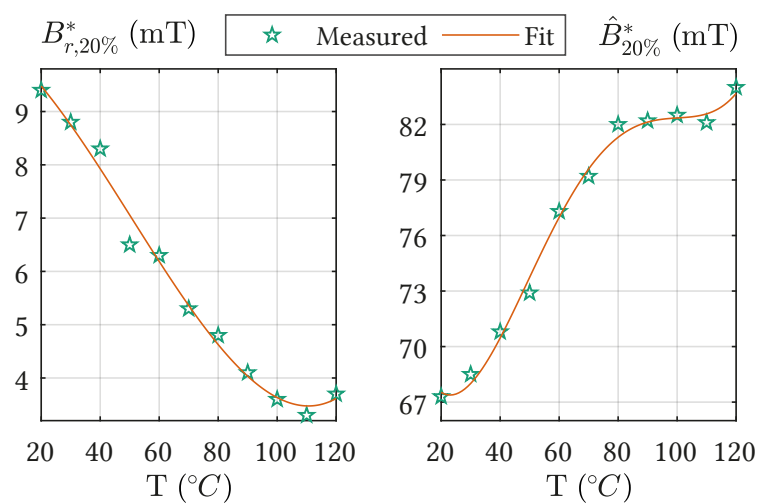

(b)
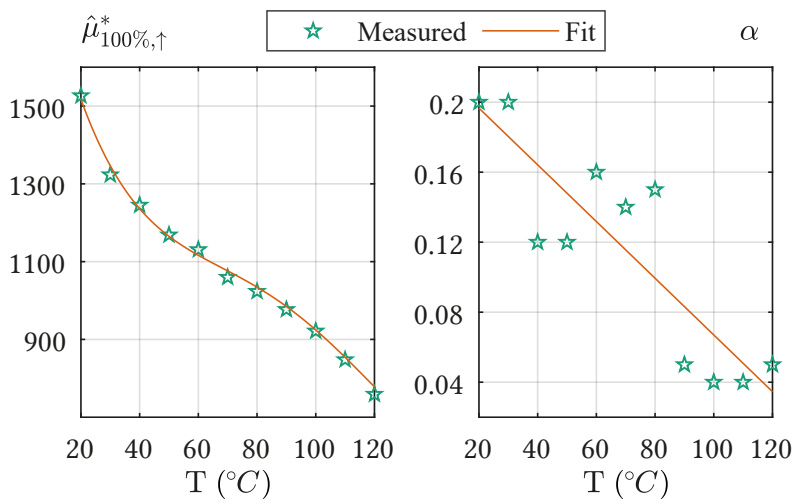

(c)

Fig. 7. Fitted parameters of the measured primary set $\left\{B_{r, 100 \%}^{*}, \hat{B}_{100 \%}^{*}\right.$ ' $\left.\hat{\mu}_{100 \%, \uparrow}^{*}, B_{r, 20 \%}^{*}, \hat{B}_{20 \%}^{*}\right\}$ and the manually selected parameter $\alpha$ in a temperature range of $\left[20^{\circ} \mathrm{C}, 120^{\circ} \mathrm{C}\right]$

sure that all the four measured temperatures lie within $\pm 0.5^{\circ} \mathrm{C}$ around the desired temperature point. Note that the core loss generated during the operation of the test sample is so small $\left(0.9 \mathrm{~W}\right.$ at $20^{\circ} \mathrm{C}$, which decreases to $0.4 \mathrm{~W}$ at $\left.120^{\circ} \mathrm{C}\right)$ that it does not contribute to the overall temperature rise of the core. To provide a relevance comparison, according to thermal measurements performed on the MFT prototype, an estimated core loss of $150 \mathrm{~W}$ is able to increase the core temperature for approximately $50^{\circ} \mathrm{C}$. The considered temperature range is limited to $\left[20^{\circ} \mathrm{C}, 120^{\circ} \mathrm{C}\right]$, due to the fact that above this

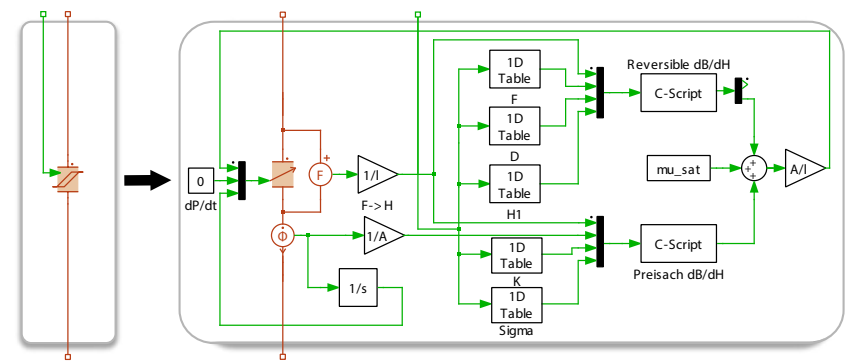

Fig. 8. Model structure of the thermally-compensated permeance block.

temperature the conductivity of the ferrite material raises. This implies an increase in core losses, since the eddy current losses gain importance and increase in value. From that point on, the used magnetic-hysteresis model, which focuses only on the frequency-independent hysteresis effect, stops being reliable. A work performed in [16] shows a similar trend in ferrite core loss elevation with increasing temperatures above $100^{\circ} \mathrm{C}$.

As described in Section III-A, in order to determine the reversible and the irreversible part of the flux density, i.e. of the permeability, the primary set of parameters extracted from the measured limiting and minor hysteresis loops is used to calculate the secondary set of coefficients $K, \sigma, F, H_{1}$, and $D$. The strong thermal influence on the primary set of parameters which can be observed in Table III for six selected temperatures is transferred over to the secondary set of parameters as well. Note that extracted values given in Table III are core-shape and material specific. Thereby, Fig. 7 shows the achieved fits of the extracted parameters in the $\left[20^{\circ} \mathrm{C}, 120^{\circ} \mathrm{C}\right]$ temperature range with $10^{\circ} \mathrm{C}$ step, i.e. 11 different temperature points. Considering the fact that the hysteresis loops measured at a single temperature point do not exactly overlap, the measured curves shown in Fig. 9 are actually envelopes of multiple hysteresis loops. The approximate thickness of the envelope in the vertical direction is $0.2 \mathrm{mT}$, whereas in the horizontal direction the thickness is estimated to $50 \mathrm{~mA} \mathrm{~m}^{-1}$. This helps in setting the expected accuracy of the extracted parameters to $\pm 0.2 \mathrm{mT}$ for parameters $\left\{B_{r, 100 \%}^{*}, \hat{B}_{100 \%}^{*}\right.$, $\left.B_{r, 20 \%}^{*}, \hat{B}_{20 \%}^{*}\right\}$. Parameter $\hat{\mu}_{100 \%, \uparrow}^{*}$ is determined with the help of the equation

$$
\hat{\mu}_{100 \%, \uparrow}^{*}=\frac{\hat{B}_{100 \%}^{*}-\hat{B}^{*}}{\left(\hat{H}_{100 \%}^{*}-\hat{H}^{*}\right) \mu_{0}} .
$$

Thereby, $\hat{B}^{*}$ and $\hat{H}^{*}$ are the values of magnetic flux density and the corresponding field strength selected from the same hysteresis loop as the maximal values $\hat{B}_{100 \%}^{*}$ and $\hat{H}_{100 \%}^{*}$, from the ascending curve in the close proximity of $\hat{B}_{100 \%}^{*}$. Due to the specific way the last parameter is calculated according to (12), parameter $\hat{\mu}_{100 \%, \uparrow}^{*}$ is considered to be accurately determined. Nevertheless, the introduced error analysis does not consider random measurement errors of the equipment. The fit functions are expressed by polynomials of the fourth order for the extracted parameters and the second order for the parameter $\alpha$. The increased deviation between the fitted function and the chosen values for $\alpha$ compared to other parameters is due to the fact that $\alpha$ value is not extracted 


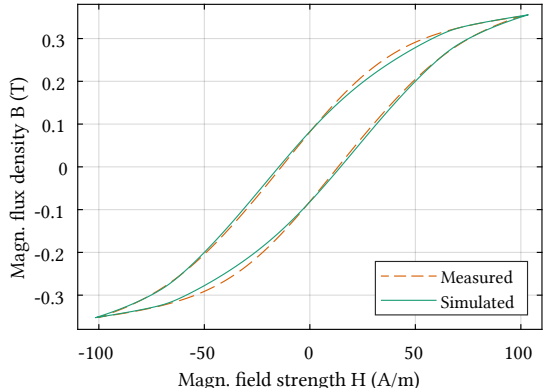

(a)

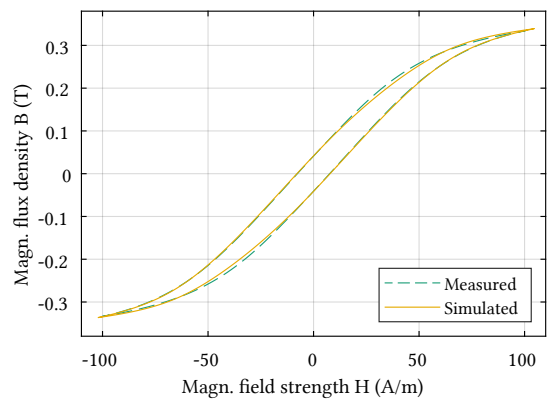

(d)

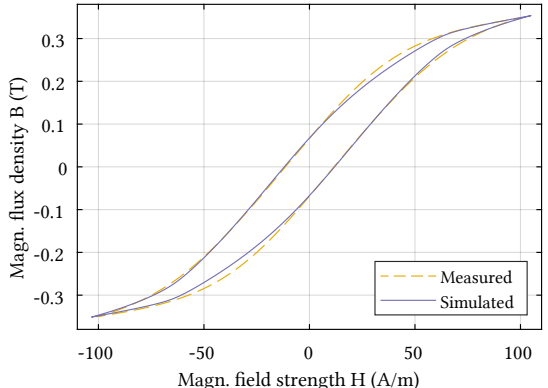

(b)

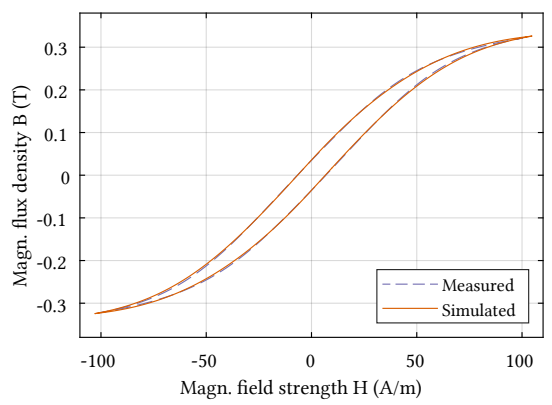

(e)

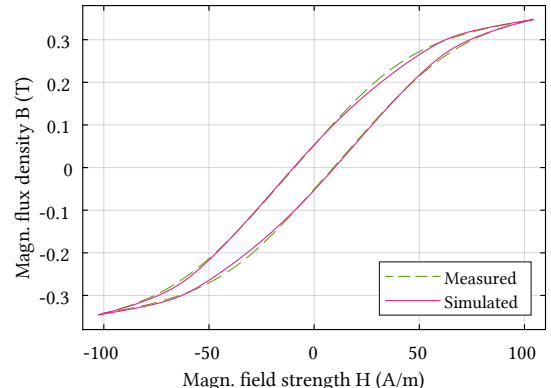

(c)

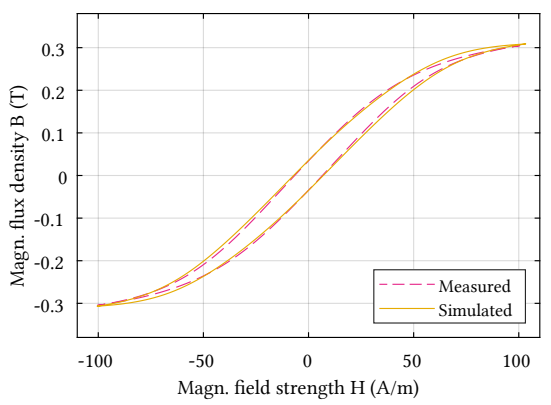

(f)

Fig. 9. Measured and simulated limiting hysteresis loops (at $\hat{H}_{100 \%}=100 \mathrm{~A} \mathrm{~m}^{-1}$ ) of the ferrite material $\mathrm{CF} 139$ at various temperatures: (a) $20^{\circ} \mathrm{C}$, (b) $40^{\circ} \mathrm{C}$, (c) $60^{\circ} \mathrm{C}$, (d) $80^{\circ} \mathrm{C}$, (e) $100^{\circ} \mathrm{C}$, (f) $120^{\circ} \mathrm{C}$.

from a hysteresis loop, but rather selected manually in order to achieve a better matching between the measured and the simulated loop. Nevertheless, the fitted function does not impair the matching excessively. Consequently, the exact temperature dependence of the secondary parameter set is considered in the simulation with the help of 1D look-up tables and by setting these parameters as the new inputs of the corresponding $\mathrm{C}$ scripts. Fig. 8 shows the implementation of the thermallycompensated permeance block, which compared to the noncompensated version requires the core temperature as its input (side input signal with arrow into the permeance block on the left). Parameters $K$ and $\sigma$ are the new inputs of the C-script calculating the irreversible permeability component, whereas $F, H_{1}$, and $D$ are the input signals for the reversible permeability part. The core temperature comes as the output of a simplified thermal model of the MFT, which is introduced in Section V.

Fig. 9 shows the matching between the measured and the simulated limiting hysteresis curves at six different temperatures. As the temperature increases, a shrinking trend of the loops is observed in both the $\mathrm{x}$ and $\mathrm{y}$ direction, which results in lower core losses. This is further confirmed in the simulations of the LLC converter shown in Section VI. Furthermore, such trend is supported by the declining values of the remanence and the saturation flux densities of the measured limiting loops for increasing temperatures found in Table III. In addition, the deviations of the per-cycle energy between the measured and the simulated hysteresis loops for each of the selected temperatures can be found in the same table. As can be observed the deviation percentage stays limited to below $10 \%$.

\section{Winding Losses}

In addition to core losses being temperature dependent, the same holds for the transformer windings as well. At higher operating frequencies the skin and proximity effect become pronounced when solid wires are used for current conduction, which leads to higher winding losses and uneven current distribution in the conductor. To prevent this and lower the ac resistance, Litz wires are commonly employed. In case of the selected MFT prototype, a square profiled copper Litz wire with 1400 strands (AWG 32) was used. For accurate estimation of winding losses a variation of the Dowell's model for foil windings from [31] was used. It yields a frequency-dependent expression for the ac winding resistance by adopting a resistance factor $F_{\mathrm{r}}$, which allows consideration of higher current harmonics. To enable the application of the Dowell's theory from foil conductors to Litz wires a porosity factor $\eta$ was introduced. Its purpose is to ensure an equal magnetic field distribution along any enclosed path for the two winding types and to assure the correct dc resistance. Therefore, the porosity factor is defined as the ratio of the actual layer copper area of the Litz wire to the effective foil conductor area as it is given by the following relation

$$
\eta=\frac{m_{\mathrm{v}} d_{\mathrm{eq}}}{H_{\mathrm{w}}} \quad \text { with } \quad d_{\mathrm{eq}}=d \sqrt{\frac{\pi}{4}} .
$$

As can be seen in Fig. 10, the parameter $m_{\mathrm{v}}$ gives the equivalent number of vertical Litz layers in a winding, $d$ is the diameter of a single Litz strand and $d_{\mathrm{eq}}$ gives the equivalent diameter of the squared strand and the thickness of the foil winding. The parameter $H_{\mathrm{w}}$ describes the height of the core window area. Furthermore, the figure shows the 

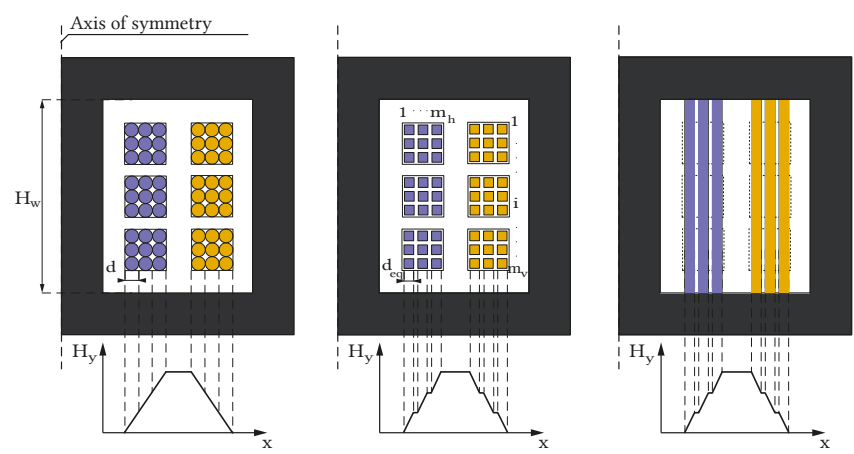

Fig. 10. Winding equivalence with magnetic field distribution: (left) Squared Litz wire windings with round strands used in the MFT prototype; (middle) equivalent squared Litz wires with square strands; (right) equivalent foil conductors which cover the full height of the core window area.

required adaptation of the winding structure, from the original MFT windings (left) to the equivalent foil conductors (right) with the corresponding magnetic field distributions in the $x$ direction. The power loss generated by conducting the current $I$ is defined by the expression

$$
\begin{gathered}
P_{\mathrm{win}}=\underbrace{\frac{\mathrm{MLT}}{\eta \sigma m_{\mathrm{h}} d_{\mathrm{eq}} H_{\mathrm{w}}}}_{R_{\mathrm{DC}}} I_{\mathrm{DC}}^{2}+\sum_{n=1}^{\infty} \underbrace{R_{\mathrm{AC}, n}}_{F_{\mathrm{r}} R_{\mathrm{DC}}} I_{\mathrm{RMS}, n}^{2} \quad \text { with } \\
F_{\mathrm{r}}=\Delta \frac{\sinh (2 \Delta)+\sin (2 \Delta)}{\cosh (2 \Delta)-\cos (2 \Delta)}+ \\
+\frac{2}{3}\left(m_{\mathrm{h}}^{2}-1\right) \Delta \frac{\sinh (\Delta)-\sin (\Delta)}{\cosh (\Delta)+\cos (\Delta)}, \quad \Delta=\frac{d_{\mathrm{eq}}}{\delta} \sqrt{\eta} .
\end{gathered}
$$

The parameter MLT is the mean length turn, $m_{\mathrm{h}}$ is the equivalent number of horizontal Litz layers, $n$ gives the harmonic current order, $\sigma$ is the copper conductivity, $\Delta$ gives the penetration ratio, whereas $\delta$ describes the skin depth.

The simulated winding losses at the temperature of $20^{\circ} \mathrm{C}$ are given in Table IV. As expected, the values of both primary and secondary winding losses rise with the increased power transfer. The exact calculation process is illustrated in Fig. 11 for the primary winding with the winding temperature and the corresponding simulated current as the inputs. Thereby, the dc and ac resistances are determined with the help of (14) and (15), respectively. Similarly, the currents in the simulation are analyzed for their dc parts and harmonic contents, which after multiplication with the corresponding resistances eventually sums up to the total winding loss. Note that no post-processing is needed in order to estimate the losses. Lastly, the winding losses of the MFT prototype reported at the full rated power in [17] differ from the simulated ones by less than $2 \%$. The reported total winding loss value is obtained based on

TABLE IV: Primary, secondary and total winding losses acquired through simulations at variable transferred power and a temperature of $20^{\circ} \mathrm{C}$.

\begin{tabular}{ccccccc}
\hline$P_{\mathrm{n}}$ & $0 \%$ & $20 \%$ & $40 \%$ & $60 \%$ & $80 \%$ & $100 \%$ \\
\hline$P_{\text {win }, 1}(\mathrm{~W})$ & 0.5 & 3 & 11.3 & 25.6 & 46.1 & 72.6 \\
\hline$P_{\text {win }, 2}(\mathrm{~W})$ & 0 & 2.9 & 11.8 & 26.7 & 47.7 & 74.9 \\
\hline$P_{\text {win,total }}(\mathrm{W})$ & 0.5 & 5.9 & 23.1 & 52.3 & 93.7 & 147.5 \\
\hline
\end{tabular}

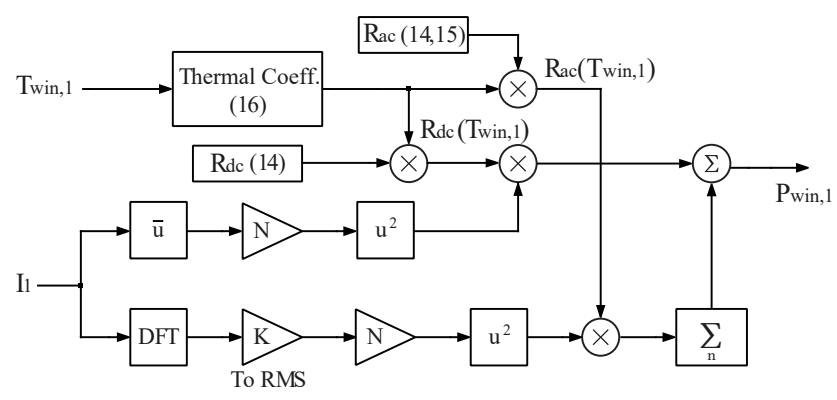

Fig. 11. Calculation scheme for the primary and secondary winding losses.

the nominal effective current and measured ac resistance at the operating frequency. Thereby, the temperature was not explicitly taken into account.

\section{Thermal Extension of the Winding Loss Model}

Temperature influence on the copper conductor losses can be taken into account with the following standard equation

$$
R_{\mathrm{xc}}\left(T_{\mathrm{win}, 1}\right)=R_{\mathrm{xc}}\left(1+\alpha_{\mathrm{Cu}}\left(T_{\mathrm{win}, 1}-T_{0}\right)\right) .
$$

Thereby, $x c$ stands for either ac or dc resistance, the variable $T_{\text {win, } 1}$ gives the simulated primary winding temperature, the parameter $\alpha_{\mathrm{Cu}}$ is the copper temperature coefficient, whereas the $T_{0}$ is the temperature at which the resistance $R_{\mathrm{xc}}$ is determined. In Fig. 11, the thermal extension of the winding model is considered in the Thermal Coeff. block where the expression from (16) is implemented. Considering the fact that the primary winding temperature $T_{\text {win, } 1}$ is influenced by the existing cooling options of the MFT as well as by the losses of the core and the secondary winding, the simulated temperature is obtained as one of the outputs of a simplified thermal MFT model, which is described in more detail in Section IV.

\section{E. Power Semiconductor Losses}

For the sake of completeness of Section III, the semiconductor losses generated during converter operation are shown in this part. It was considered that the switching devices are

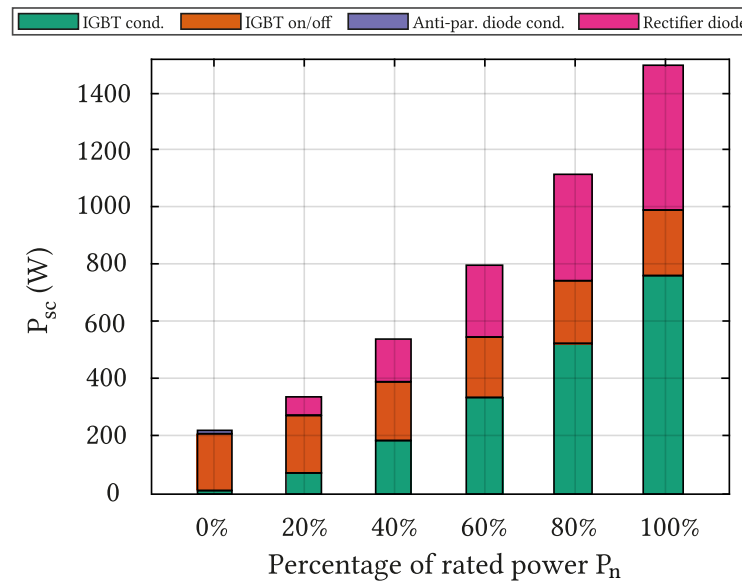

Fig. 12. Semiconductor losses calculated for various output powers with the magnetic hysteresis model of the MFT operated at $10 \mathrm{kHz}$. 
spatially distant from the MFT, so that the semiconductor losses do not affect the transformer losses and vice versa. For simulation purposes of the power stages, the IGBT module 5SNG 0150Q170300 from ABB is selected as a switching device. The corresponding thermal descriptions of the IGBT and the anti-parallel diode are found online [32] and are directly included in the simulation, since the loss tool of PLECS is intrinsically capable of performing thermal compensation of this type of losses. Note that this is not the case with magnetic components. The descriptions contain device's switching energies and static characteristics for several temperatures (usually $25^{\circ} \mathrm{C}, 125^{\circ} \mathrm{C}$ and $175^{\circ} \mathrm{C}$ ). Thanks to an available feedback of the junction temperature, it is possible to determine the losses of the device within the simulation considering specific thermal conditions. Therefore, the determination of semiconductor losses will not be considered in more detail. Fig. 12 shows the estimated losses of all the switching devices in the inverter and rectifier power stage simulated for various loading conditions from no load to full load. Thereby, the transformer is modeled with the help of the magnetic-hysteresis model.

\section{Thermal Modeling of The MFT}

In power electronics applications, the thermal time constants are usually several magnitudes of order higher compared to the electrical ones. As a consequence, in a joint electrical-thermal simulation the computational effort, required to respect both long simulation times due to thermal processes and small enough time steps needed for solving electromagnetic equations, tends to be enormous. In order to reduce it to a certain extent, only a simplified thermal model of the transformer is considered in order to show the functionality of the thermallycompensated magnetic model. The simplification is based on the static thermal model [19] which is specifically developed for the considered MFT prototype. Note that the focus of this paper is not on establishing a novel MFT thermal model, but to show that it is possible to close a thermal loop of a magnetic component inside of a time-domain simulation.

In general, there are three main heat exchange mechanisms, namely, conduction, convection and radiation. To simplify the thermal model of the MFT as much as possible, the thermal resistances corresponding to each of these mechanisms, and assigned to interfaces between different parts of the core and the windings, as well as to interfaces towards the ambient are aggregated into six thermal resistances. Thereby, the core is represented uniformly by a single thermal capacitor. Similarly, the primary and secondary windings are both assigned a thermal capacitance value based on the utilized amount of copper and the specific heat capacity. Thermal interfaces between the core and each of the windings, as well as between the primary and the secondary winding, and the three transformer components (core and two windings) and

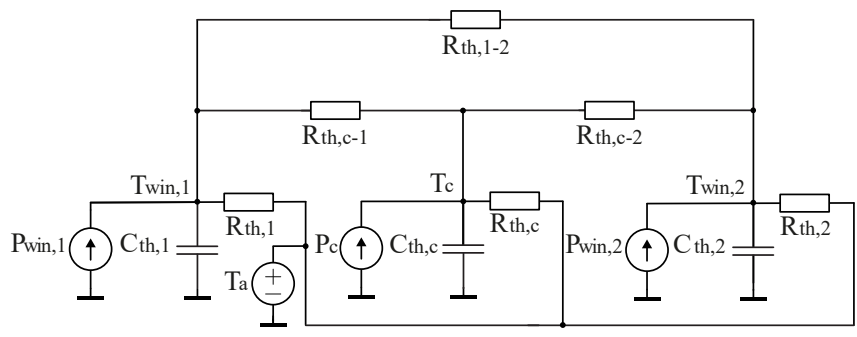

Fig. 13. Simplified thermal model of the MFT, $T_{\mathrm{a}}=20^{\circ} \mathrm{C}$.

the surrounding ambient are represented by certain values of thermal resistances. Some of these values are estimated based on thermal and loss measurements taken during a nine-hour thermal run performed with the considered MFT prototype [17] with the help of the equation $R_{\mathrm{th}}=\frac{P_{\text {loss }}}{\Delta T}$. The values of each of the resistances portray the existing cooling conditions. The simplified thermal model of the MFT can be seen in Fig. 13. Thereby, the transformer losses are included through current sources. Nevertheless, to increase the simulation speed all three thermal capacitances are set to small values compared to the initial ones, so that a steady-state temperature can be reached fast, i.e. in several seconds instead of several hours, which is the case in reality. Therefore, some of the values are set by manual adjustments so that a steady-state can be achieved in the selected simulation time of $3 \mathrm{~s}$. Moreover, the average values of the steady-state temperatures are mainly governed by the thermal resistances.

Furthermore, the MFT is constructed in a way that the primary winding lies closer to the middle limb of the core, whereas the secondary winding encircles it. Thereby, the primary winding has a smaller MLT, yet a higher thermal resistance compared to the secondary winding, since it is being heated predominantly through convection mechanism but also through radiation from both sides, by the core and by the secondary winding. Table $\mathrm{V}$ gives an overview of the simulated and estimated values of thermal parameters of the model. Based on the existing thermal measurements, it was not possible to estimate the values of thermal resistances which would describe thermal processes occurring on the interfaces between the core and the windings. For those reasons, the three thermal resistances $R_{\mathrm{th}, 1-2}, R_{\mathrm{th}, \mathrm{c}-1}, R_{\mathrm{th}, \mathrm{c}-2}$ are selected to be in a similar range to the estimated values.

\section{Simulation Results}

To demonstrate the effectiveness and the capability of the introduced thermally-compensated magnetic hysteresis model two simulations of the LLC converter circuit shown in Fig. 1 are run in parallel. Namely, one simulation is performed without the thermal compensation, whereas the other is run with included thermal extensions of both core and winding

TABLE V: Thermal parameters of the simplified thermal model of the MFT.

\begin{tabular}{lccccccccc}
\hline Parameter & $R_{\mathrm{th}, \mathrm{c}}(\mathrm{W} / \mathrm{K})$ & $C_{\mathrm{th}, \mathrm{c}}(\mathrm{J} / \mathrm{K})$ & $R_{\mathrm{th}, 1}(\mathrm{~W} / \mathrm{K})$ & $C_{\mathrm{th}, 1}(\mathrm{~J} / \mathrm{K})$ & $R_{\mathrm{th}, 2}(\mathrm{~W} / \mathrm{K})$ & $C_{\mathrm{th}, 2}(\mathrm{~J} / \mathrm{K})$ & $R_{\mathrm{th}, 1-2}(\mathrm{~W} / \mathrm{K})$ & $R_{\mathrm{th}, \mathrm{c}-1}(\mathrm{~W} / \mathrm{K})$ & $R_{\mathrm{th}, \mathrm{c}-2}(\mathrm{~W} / \mathrm{K})$ \\
\hline Estimated & 2.5 & 15360 & 1.6 & 92.9 & 0.8 & 92.9 & - & - \\
\hline Simulated & 1.15 & 1.25 & 1.04 & 0.125 & 0.8 & 0.125 & 1.3 & 1.56 \\
\hline
\end{tabular}


loss models. Furthermore, both simulations are following the same power load profile and the electrical parameters given in Tab. I are kept the same. Finally, this section compares the obtained simulation results. The exact implementation of thermal compensation of transformer core and winding losses in a simulation environment is introduced in Sections III-B and III-D. As assumed earlier, the transformer losses have no impact on the switching devices, therefore, the simulated primary and secondary MFT currents retain the same shape and values regardless of the thermal extension. Fig. 14 reveals the transformer currents after a gradual power load change (visible from the first plot) has been applied in the simulation. Namely, a step to $50 \%$ of the full power load $\left(P_{\mathrm{n}}=100 \mathrm{~kW}\right)$ is introduced at the time point of $0.9 \mathrm{~s}$, followed by the step to full power load at $1.9 \mathrm{~s}$. The times are selected in a way to allow the temperatures and losses to reach a steady state. Before the first step to $50 \mathrm{~kW}$, no power has been transfered through the converter. Nevertheless, in order to keep the transformer core magnetized the magnetizing current is required, which results in certain semiconductor, core and primary winding losses (cf. Fig. 15). The second plot of Fig. 14 shows the envelopes of the primary and the magnetizing current with an enlarged view of a single period of each current, simulated after steady state has been reached for full power transfer. In a similar fashion, the last plot in the figure gives the secondary transformer current. The enlarged plots show current waveforms characteristic for the HC-DCM operation mode of the LLC converter, which can be clearly recognized in the shape of the secondary transformer current.

Left-hand side of Fig. 15, i.e. Figs. 15a and 15c show simulated hysteresis curves, as well as loss and temperature progressions of the transformer core and windings during a simulation time of $3 \mathrm{~s}$ in case no thermal compensation of the hysteresis and the winding model is undertaken. Similarly, Figs. $15 \mathrm{~b}$ and $15 \mathrm{~d}$ provide simulation results when thermal compensation is included. Tab. VI provides an overview of the simulated steady-state power loss and temperature values for the core and the windings depending whether thermal compensation is included in the simulation or not.

To begin with, Figs. $15 \mathrm{a}$ and $15 \mathrm{~b}$ show the hysteresis curve at two time occasions for multiple periods, that is at the beginning (at $0.5 \mathrm{~s}$ ) and towards the end of the simulation (at $2.5 \mathrm{~s}$ ). Thereby, it is clear from Fig. 15b that with thermal extension present in the simulation, the hysteresis loop shrinks and changes with temperature rise, as expected and visualized in Fig. 9 in Section III-B. Moreover, a smeared hysteresis curve, i.e. envelope visible in Fig. 15b corresponds to the fact that the hysteresis loop gets adapted for every new degree Celsius that the estimation of the core temperature, given by

TABLE VI: Simulated power loss and temperature values of the core and the windings in steady-state with and without thermal compensation of the magnetic hysteresis and the winding model.

\begin{tabular}{ccccccc}
\hline Steady-state values & $P_{\mathrm{c}}$ & $T_{\mathrm{c}}$ & $P_{\text {win, } 1}$ & $T_{\text {win }, 1}$ & $P_{\text {win }, 2}$ & $T_{\text {win }, 2}$ \\
\hline No thermal comp. & $216 \mathrm{~W}$ & $163^{\circ} \mathrm{C}$ & $72 \mathrm{~W}$ & $121{ }^{\circ} \mathrm{C}$ & $75 \mathrm{~W}$ & $120^{\circ} \mathrm{C}$ \\
\hline Thermal comp. & $69 \mathrm{~W}$ & $100^{\circ} \mathrm{C}$ & $97 \mathrm{~W}$ & $109^{\circ} \mathrm{C}$ & $99 \mathrm{~W}$ & $102{ }^{\circ} \mathrm{C}$ \\
\hline
\end{tabular}

the simplified thermal model of the MFT, changes. This is further visible in both core temperature and core losses curves shown in Fig. 15d. That is to say that with the generated core and winding losses the transformer structure heats up, which, due to specificity of the ferrite material, implies shrinking of the corresponding hysteresis loop and subsequent reduction in core losses until the point where a steady-state is reached. According to Tab. VI, core losses reduce to a value of $69 \mathrm{~W}$ at a stationary temperature of $100^{\circ} \mathrm{C}$, compared to a core temperature of $163^{\circ} \mathrm{C}$ and a power loss of $216 \mathrm{~W}$ which are obtained without thermal compensation in the simulation. As expected for the thermally non-compensated hysteresis model, the core temperature change visible in Fig. 15c does not effect the core losses. This is visible from the upper plot of the same figure, where the core loss curve reaches fast a steadystate value after each of the introduced power steps. Moreover, a slight reduction in core losses with the increase of the transferred power is in accordance with Tab. II. Spikes in core loss values visible after each of the steps in the power load for both simulation cases is due to the necessary time for the magnetic hysteresis model to reach its convergence after the operating point has been changed. It is not to be confused with the convergence and steady state of the simplified thermal model.

Note that the simulated semiconductor losses shown in Fig. 15 are scaled differently, ten times smaller, for presentation purposes. As expected, these losses remain the same regardless of thermal compensation, and they develop according to the load profile. Furthermore, due to a positive thermal coefficient of copper and according to (16), an increase in winding losses in case of thermal extension of the winding model $\left(P_{\text {win, } 1} \approx 97 \mathrm{~W}\right)$ is reasonable and anticipated compared to the non-compensated simulation results $\left(P_{\text {win, } 1} \approx 72 \mathrm{~W}\right)$. This can be observed in Fig. 15d and in Tab. VI based on the reached stationary winding loss values. Nevertheless, due to decreased core temperature and reduced core losses, the steady-state winding temperatures $\left(T_{\text {win, } 1} \approx 109^{\circ} \mathrm{C}\right.$, $T_{\text {win }, 2} \approx 102^{\circ} \mathrm{C}$ ) simulated in case of thermal compensation are lower than the ones simulated without the thermal compensation $\left(T_{\text {win }, 1} \approx 121^{\circ} \mathrm{C}, T_{\text {win }, 2} \approx 120^{\circ} \mathrm{C}\right.$ ). Compared to the secondary winding, a higher primary winding temperature is explained by its position in the MFT prototype structure. Lastly, a staircase alike progression of temperatures and losses for both windings is observed due to the selected power load profile.

\section{Vi. Conclusion}

In this paper, a thermally-compensated magnetic hysteresis model was introduced and its implementation in a simulation environment was presented in a comprehensive manner. Moreover, the modeling of the winding losses based on the Dowell's model was thermally extended as well. In this way, the main transformer components are capable of considering the corresponding temperatures for loss estimation, which finally closes a thermal loop for this magnetic component. A simplification of an existing static thermal model of the MFT was developed in order to provide estimates of the core 


\section{IEEE TRANSACTIONS ON POWER ELECTRONICS}

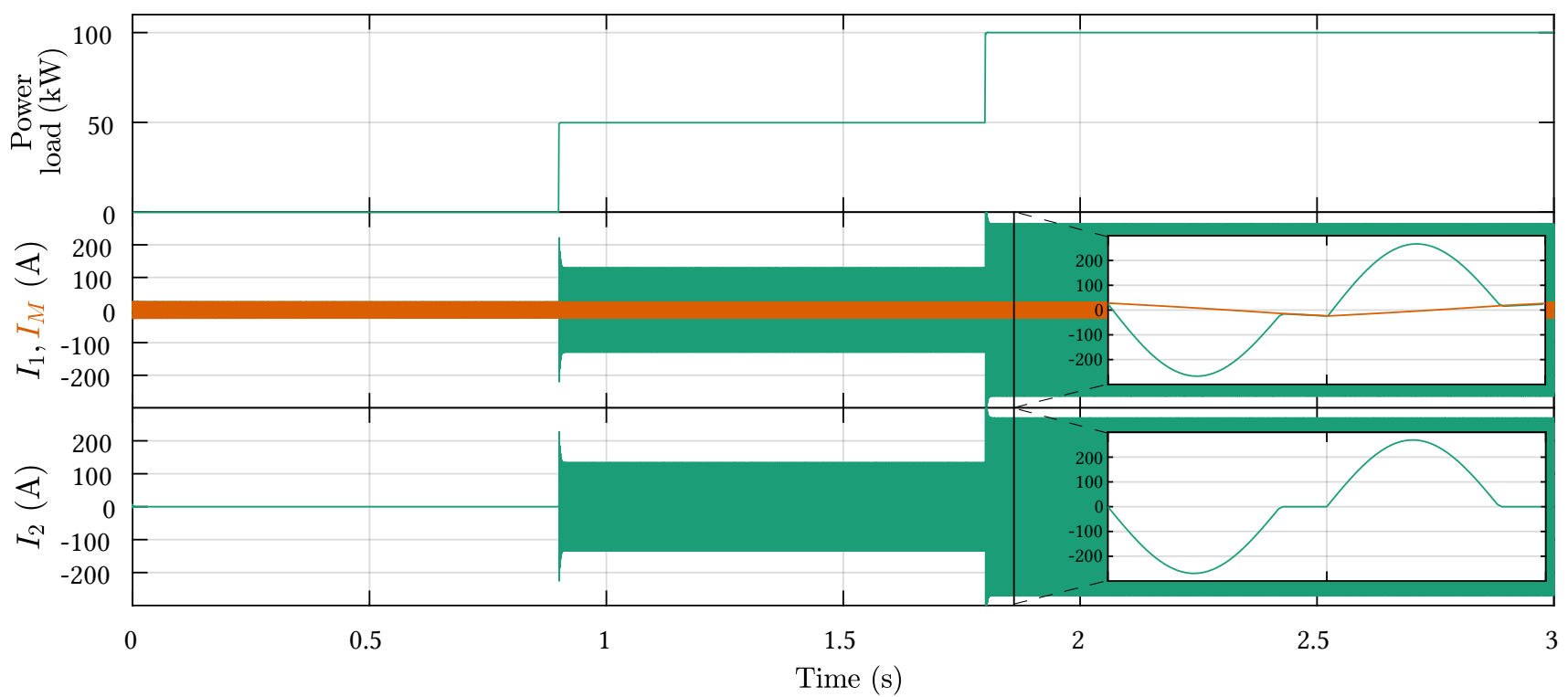

Fig. 14. Transformer currents (middle and bottom plot) simulated for the selected case study for a power load change in steps (upper plot).



(a)

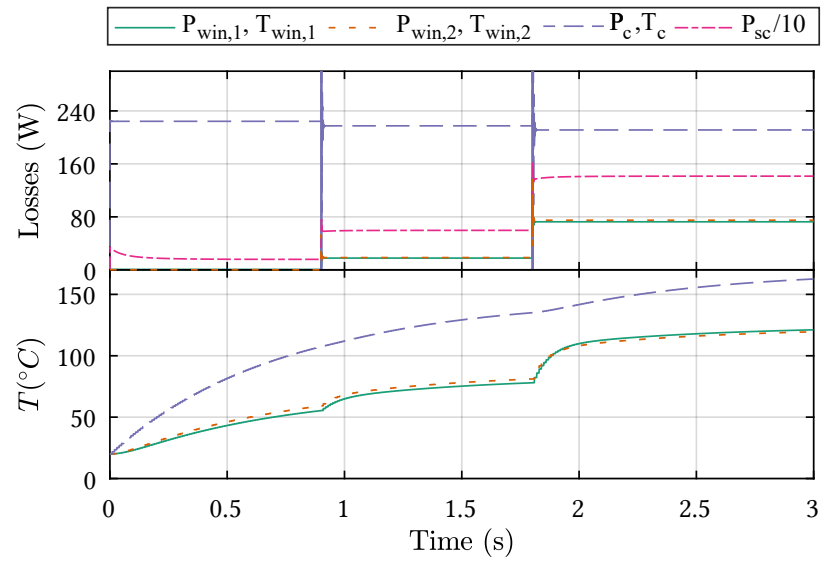

(c)



(b)



(d)

Fig. 15. Simulated hysteresis loops for thermally (a) non-compensated and (b) compensated MFT model. Power losses and temperature progressions of the core and the windings simulated with (c) the non-compensated and (d) thermally-compensated hysteresis and winding model. 
and winding temperatures. The presented simulation results prove the capability of the introduced thermal extension of the magnetic hysteresis model to take the estimated core temperature into account and based on it adjust the modeling of the hysteresis loop. This approach provides a new degree of freedom to improve the model accuracy. For the selected case study, the steady-state power losses of the MFT core are reduced by $68 \%$, whereas the corresponding temperature is adjusted to a $39 \%$ lower value. With the correct parametrization of the thermal MFT model, the introduced thermallycompensated magnetic hysteresis model has a great potential of improving the existing core loss estimation techniques.

\section{ACKNOWLEDGMENT}

The results presented in this paper are a part of the EMPOWER project that has received funding from the European Research Council (ERC) under the European Union's Horizon 2020 research and innovation programme (Grant agreement No. 818706).

\section{REFERENCES}

[1] M. K. Kazimierczuk and D. Czarkowski, Resonant power converters. John Wiley \& Sons, 2012

[2] R. W. De Doncker, D. M. Divan, and M. H. Kheraluwala, "A threephase soft-switched high-power-density dc/dc converter for high-power applications," IEEE transactions on industry applications, vol. 27, no. 1, pp. 63-73, 1991

[3] J. B. Goodenough, "Summary of losses in magnetic materials," IEEE Transactions on magnetics, vol. 38, no. 5, pp. 3398-3408, 2002.

[4] M. L. Hodgdon, "Mathematical theory and calculations of magnetic hysteresis curves," IEEE Transactions on Magnetics, vol. 24, no. 6 , pp. 3120-3122, 1988

[5] L. Chua and K. Stromsmoe, "Lumped-circuit models for nonlinear inductors exhibiting hysteresis loops," IEEE Transactions on Circuit Theory, vol. 17, no. 4, pp. 564-574, 1970.

[6] D. C. Jiles and D. L. Atherton, "Theory of ferromagnetic hysteresis," Journal of magnetism and magnetic materials, vol. 61, no. 1-2, pp. 4860, 1986

[7] D. C. Jiles and J. Thoelke, "Theory of ferromagnetic hysteresis: Determination of model parameters from experimental hysteresis loops," IEEE Transactions on magnetics, vol. 25, no. 5, pp. 3928-3930, 1989.

[8] F. Preisach, "Über die magnetische Nachwirkung," Zeitschrift für Physik, vol. 94, no. 5-6, pp. 277-302, 1935

[9] A. Benabou, S. Clénet, and F. Piriou, "Comparison of preisach and jiles-atherton models to take into account hysteresis phenomenon for finite element analysis," Journal of magnetism and magnetic materials, vol. 261, no. 1-2, pp. 139-160, 2003.

[10] I. Villar, "Multiphysical characterization of medium-frequency power electronic transformers," PhD thesis, EPFL, 2010.

[11] M. Luo, D. Dujic, and J. Allmeling, "Modeling frequency independen hysteresis effects of ferrite core materials using permeance-capacitance analogy for system-level circuit simulations," IEEE Transactions on Power Electronics, vol. 33, no. 12, pp. 10 055-10 070, 2018.

[12] J. H. Chan, A. Vladimirescu, X.-C. Gao, P. Liebmann, and J. Valainis, "Nonlinear transformer model for circuit simulation," IEEE Transactions on Computer-Aided Design of Integrated Circuits and Systems, vol. 10 , no. 4, pp. 476-482, 1991

[13] M. Kachniarz, J. Salach, R. Szewczyk, and A. Bieńkowski, "Temperature influence on the magnetic characteristics of mn-zn ferrite materials," in Progress in Automation, Robotics and Measuring Techniques, Springer, 2015, pp. 121-127.

[14] B. Ahmed, J. Ahmed, and G. Guy, "Computing ferrite core losses at high frequency by finite elements method including temperature influence," IEEE transactions on magnetics, vol. 30, no. 5, pp. 3733 3736, 1994.

[15] R. Chen, Z. Dong, Z. Zhang, H. Gui, J. Niu, R. Ren, F. Wang, L. M. Tolbert, B. J. Blalock, D. J. Costinett, et al., "Core characterization and inductor design investigation at low temperature," in 2018 IEEE Energy Conversion Congress and Exposition (ECCE), IEEE, 2018, pp. 42184225
[16] M. Yang, Y. Li, Q. Yang, Z. Lin, S. Yue, H. Wang, and C. Liu, "Magnetic properties measurement and analysis of high frequency core materials considering temperature effect," IEEE Transactions on Applied Superconductivity, vol. 30, no. 4, pp. 1-5, 2020.

[17] M. Mogorovic and D. Dujic, "100 kw, $10 \mathrm{khz}$ medium-frequency transformer design optimization and experimental verification," IEEE Transactions on Power Electronics, vol. 34, no. 2, pp. 1696-1708, 2018

[18] M. Luo, D. Dujic, and J. Allmeling, "Modeling frequency-dependent core loss of ferrite materials using permeance-capacitance analogy for system-level circuit simulations," IEEE Transactions on Power Electronics, vol. 34, no. 4, pp. 3658-3676, 2018.

[19] M. Mogorovic and D. Dujic, "Thermal modeling and experimental verification of an air cooled medium frequency transformer," in 2017 19th European Conference on Power Electronics and Applications (EPE'17 ECCE Europe), IEEE, 2017.

[20] J. Muehlethaler, J. Biela, J. W. Kolar, and A. Ecklebe, "Improved coreloss calculation for magnetic components employed in power electronic systems," in IEEE Transactions on Power Electronics, vol. 27, 2012 pp. 964-973.

[21] M. Luo, "Dynamic modeling of magnetic components for circuit simulation of power electronic systems," PhD thesis, EPFL, 2018.

[22] M. Luo, D. Dujic, and J. Allmeling, "Test setup for characterisation of biased magnetic hysteresis loops in power electronic applications," in 2018 International Power Electronics Conference (IPEC-Niigata 2018ECCE Asia), IEEE, 2018, pp. 422-427.

[23] E. Della Torre, Magnetic hysteresis. Wiley, 2000.

[24] C. P. Steinmetz, "On the law of hysteresis," Proceedings of the IEEE, vol. 72, no. 2, pp. 197-221, 1984

[25] K. Venkatachalam, C. R. Sullivan, T. Abdallah, and H. Tacca, "Accurate prediction of ferrite core loss with nonsinusoidal waveforms using only steinmetz parameters," in 2002 IEEE Workshop on Computers in Power Electronics, 2002. Proceedings., IEEE, 2002.

[26] N. Djekanovic, M. Luo, and D. Dujic, "Integrated simulation approach to loss calculations of power converter systems," in PCIM Europe 2020; International Exhibition and Conference for Power Electronics Intelligent Motion, Renewable Energy and Energy Management, VDE, 2020, pp. 1-8.

[27] H. Y. Lu, J. G. Zhu, and S. R. Hui, "Measurement and modeling of thermal effects on magnetic hysteresis of soft ferrites," IEEE Transactions on Magnetics, vol. 43, no. 11, pp. 3952-3960, 2007.

[28] J. Y. Alsawalhi and S. D. Sudhoff, "Saturable thermally-representative steinmetz-based loss models," IEEE transactions on magnetics, vol. 49 no. 11 , pp. 5438-5445, 2013.

[29] J. Reinert, A. Brockmeyer, and R. W. De Doncker, "Calculation of losses in ferro-and ferrimagnetic materials based on the modified steinmetz equation," IEEE Transactions on Industry applications, vol. 37, no. 4, pp. 1055-1061, 2001.

[30] J. Li, T. Abdallah, and C. R. Sullivan, "Improved calculation of core loss with nonsinusoidal waveforms," in Conference Record of the 2001 IEEE Industry Applications Conference. 36th IAS Annual Meeting (Cat. No. 01CH37248), IEEE, vol. 4, 2001, pp. 2203-2210.

[31] P. Dowell, "Effects of eddy currents in transformer windings," Proceedings of the Institution of Electrical Engineers, vol. 113, no. 8, p. $1387,1966$.

[32] $A B B$ Semiconductors. [Online]. Available: https://www. hitachiabbpowergrids.com/es/es/offering/product-and-system/semiconductors/ insulated-gate-bipolar-transistor-igbt-and-diode-modules.

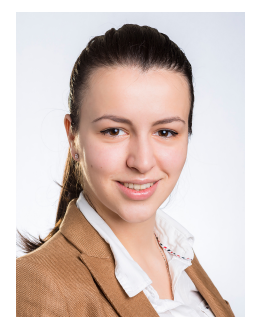

Nikolina Djekanovic received the B.Sc. and Dipl.Ing. degrees from Vienna University of Technology (TU Wien), Vienna, Austria, in 2016 and 2018, respectively. She is currently working toward the Ph.D. degree at École Polytechnique Fédérale de Lausanne (EPFL), Lausanne, Switzerland. During the M.Sc. degree, she spent one year at KTH Royal Institute of Technology, Stockholm, Sweden, as an Exchange student and a Research Engineer.

In 2019, she joined Power Electronics Laboratory, EPFL, as a Doctoral Research Assistant. Her research interests include modeling, design, and optimization of high-power medium-voltage transformers, power electronics, and batteries. She is an IEEE Student Member 


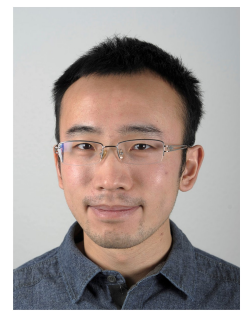

Min Luo was born in Beijing, China, in 1986. He received the B.S. degree in electrical engineering from Tsinghua University, Beijing, China, in 2009, M.S. degree in electrical power engineering from RWTH Aachen University, Aachen, Germany, in 2012, and PhD degree in electrical engineering from École Polytechnique Fédérale de Lausanne (EPFL), Switzerland, in 2018. Since 2012 he has been with Plexim as an application engineer, working on the toolchain PLECS for fast simulation of power electronic systems.

His current research interest include dynamic modelling of magnetic components and real-time HIL simulation of power converters.

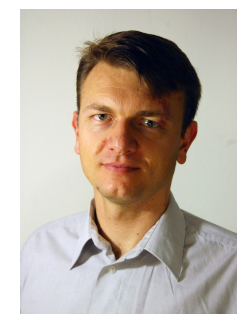

Dražen Dujić (S'03-M'09-SM'12) received the Dipl. -Ing. and M.Sc. degrees from the University of Novi Sad, Novi Sad, Serbia, in 2002 and 2005, respectively, and the Ph.D. degree from the Liverpool John Moores University, Liverpool, U.K., in 2008, all in electrical engineering.

From 2002 to 2006, he was with the Department of Electrical Engineering, University of Novi Sad as a Research assistant, and from 2006 to 2009 with Liverpool John Moores University as a Research Associate. From 2009 till 2013, he was with ABB Corporate Research Centre, Switzerland, as a Principal Scientist working on the power electronics projects spanning the range from low-voltage/power SMPS in below kilowatt range to medium voltage high-power converters in a megawatt range. From 2013 till 2014, he was with ABB Medium Voltage Drives, Turgi, Switzerland, as R\&D Platform Manager, responsible for ABB's largest IGCT based medium voltage drive ACS6000. He is currently with École Polytechnique Fédérale de Lausanne EPFL, Lausanne, Switzerland, as an Assistant Professor and the Director of the Power Electronics Laboratory. His current research interests include the areas of design and control of advanced high-power electronics systems and high performance drives. He has authored or coauthored more than 150 scientific publications and has filed 16 patents.

$\mathrm{He}$ is an Associate Editor for IEEE Transactions on Industrial Electronics, IEEE Transaction on Power Electronics and IET Electric Power Applications. In 2018 he has received EPE Outstanding Service Award, and in 2014 the Isao Takahashi Power Electronics Award for outstanding achievement in power electronics. 